\title{
High TMEM45A expression is correlated to epidermal keratinization
}

\author{
Aurélie Hayez ${ }^{1}$, Jérémy Malaisse ${ }^{1}$, Edith Roegiers ${ }^{2}$, Marie Reynier $^{3}$, Chantal Renard $^{4}$, Marek Haftek ${ }^{5}$, \\ Vincent Geenen ${ }^{4}$, Guy Serre ${ }^{3}$, Michel Simon ${ }^{3}$, Catherine Lambert de Rouvroit ${ }^{1}$, Carine Michiels ${ }^{2}$ and \\ Yves Poumay ${ }^{1}$
}

${ }^{1}$ Cell and Tissue Laboratory-URPhyM-NARILIS, University of Namur, Namur, Belgium; ${ }^{2}$ Research Unit for Cell biology-NARILIS, University of Namur, Namur, Belgium; ${ }^{3}$ UMR5165/U1056 CNRS-INSERM-University of Toulouse, UDEAR, Toulouse, France; ${ }^{4}$ Center of Immunoendocrinology, GIGA-I ${ }^{3}$, University of Liège, Liège, Belgium; ${ }^{5}$ Laboratoire de Recherche Dermatologique, University of Lyon, Lyon, France Correspondence: Yves Poumay, Cell and Tissue Laboratory-URPhyM-NARILIS, University of Namur, Rue de Bruxelles, 61, B-5000 Namur, Belgium, Tel.: + 328172 42 57, Fax: + 32817242 61, e-mail: yves.poumay@unamur.be

Abstract: TMEM45A (DERP7, DNAPTP4 or FLJ10134) gene,

belonging to the TMEM family encoding predicted transmembrane proteins, is highly expressed in epidermal keratinocytes. To investigate the potential involvement of TMEM45A during the differentiation and keratinization processes, its expression has been characterized in normal human keratinocytes and the protein subcellular localization has been studied in this cell type, both in vitro and in vivo. TMEM45A expression is upregulated with differentiation, either induced by cultured keratinocyte confluence or enhanced $\mathrm{Ca}^{2+}$ concentration in medium. In vivo, TMEM45A mRNA and protein are mostly found in the granular layer of the epidermis. TMEM45A expression is linked to keratinization, as accumulation of the protein is detected in native and reconstructed epidermis as well as in thymic Hassal bodies, but not in non-keratinized stratified epithelia. At the subcellular level, co-detection with ER and Golgi markers reveals that TM protein $45 \mathrm{~A}$ is associated with the Golgi apparatus and more specifically with the trans-Golgi/trans-Golgi network in vitro and in granular layer in vivo. The protein is neither related to lysosomes nor transported within corneodesmosin-containing lamellar bodies. These data demonstrate a strong correlation between TMEM45A expression and epidermal keratinization, indicating the relevance of this gene in this process.

Abbreviations: CALN, calnexin; CDSN, corneodesmosin; ER, endoplasmic reticulum; FLG, filaggrin; IVL, involucrin; KLK7, kallikrein 7; KRT, keratin; TM protein $45 \mathrm{~A}$, transmembrane protein $45 \mathrm{~A}$.

Key words: epidermis - Golgi apparatus - granular layer - keratinization - keratinocyte

Accepted for publication 30 March 2014

\section{Introduction}

The epidermis is a squamous stratified epithelium where keratinocytes follow a unique programme of terminal differentiation and cell death that leads to the formation of the cornified layer, the outermost skin barrier. During this so-called keratinization process, the cell content including all organelles is replaced by compacted keratin filaments. At the cytoplasmic side of plasma membrane, various proteins are enzymatically cross-linked to form a cornified envelope. Finally, modifications of lipids secreted in the intercellular spaces occur, leading to the formation of a hydrophobic matrix (1). Many keratinizing disorders in humans have been identified resulting from mutations in genes critical for keratinization (e.g. keratins, filaggrin, loricrin, SPINK5, transglutaminases), for anchoring junctions or for lipids metabolism (2-5).

Currently, our knowledge about gene expression during keratinization remains incomplete (6). However, recent transcriptomic studies allowed identification of yet unknown although potentially major actors of this process. Indeed, a transcriptomic study based on reconstructed human epidermis classified the still poorly characterized TMEM45A gene in a cluster particularly highly expressed at the late differentiation stage (7). This cluster also contains numerous genes of the epidermal differentiation complex and gathers genes implicated in ectoderm and epidermis development, morphogenesis and keratinization. Moreover, according to BioGPS (8), skin is the tissue expressing TMEM45A mRNA at the highest level.
TMEM45A gene (also called DERP7, DNAPTP4 or FLJ10134) belongs to the large family of genes encoding uncharacterized predicted transmembrane (TMEM) proteins. To date, almost all available information about TMEM45A is coming from transcriptomic studies, databases or bioinformatics predictive tools. Seven transcripts have been reported, but only three isoforms of transmembrane protein 45A (TM protein 45A) have been detected: first contains 191 amino acids with $21.9 \mathrm{kDa} \mathrm{MW}$, a second contains 275 amino acids with $31.7 \mathrm{kDa}$ MW and a third contains 291 amino acids with $33.5 \mathrm{kDa} \mathrm{MW}$, according to Ensembl (9). The isoforms contain three, five or seven predicted transmembrane domains, according to PredictProtein (10) and SOSUI (11), thus they are suspected to be integral proteins according to Uniprot (12). Subcellular localization and functions remain unknown. Recently, we briefly reported selective induction of TMEM45A expression in differentiating keratinocytes, as well as increased expression in normal human epidermis (13), data later confirmed by others (14). Finally, in an approach using keratinocytes fractions purified from normal epidermis, TMEM45A was reported as a differentiation-associated gene, because of its significantly higher mRNA level in granular and cornified keratinocytes-enriched fraction compared with its level in basal keratinocytes-enriched fraction (15).

In addition, our current knowledge about TMEM45A is also incomplete in pathological conditions. Regarding skin pathology, 
Hayez et al.

no significant variation in TMEM45A mRNA was observed between healthy skin biopsies and atopic dermatitis, basal cell carcinoma, squamous cell carcinoma or melanoma biopsies, whereas a significant upregulation was observed in psoriasis and actinic keratosis $(14,16)$. Moreover, TMEM45A with other genes involved in differentiation (e.g. FLG, LOR, KRT10) was found downregulated in cutaneous squamous cell carcinoma in comparison with actinic keratosis (17). However, how alterations of TMEM45A expression are involved in diseases remains to be solved. In cancer cells, though, TMEM45A expression is implicated in resistance against drug-induced apoptosis in hypoxic conditions by a still unknown mechanism (13). Conversely, it suppresses the progression of ductal carcinoma into invasive breast cancer, again by an unknown mechanism (18).

Here, we further characterized the expression of the new potential epidermal actor TMEM45A in vitro and in vivo to assess its particular involvement in the keratinization process. Next, we studied subcellular localization of the TM protein $45 \mathrm{~A}$ in keratinocytes to get more insight into its potential functions.

\section{Materials \& methods}

Antibodies, immunogenic peptide and chemicals

The list is available in the Supplementary Material.

Isolation of normal human keratinocytes and induction of differentiation in autocrine cultures

Primary keratinocytes were isolated from normal human adult abdominal skin collected at plastic surgery after written informed consent of patients (Dr. B. Bienfait, Clinique St Luc, Namur, Belgium). Cells were cultured in autocrine conditions as described (19). All experiments were carried out according to the Declaration of Helsinki Principles and were approved by the Medical Ethical Committee of Clinique St Luc.

Calcium-induced differentiation of keratinocytes

Keratinocytes monolayers were seeded at very low cell density $\left(5000 \mathrm{cells} / \mathrm{cm}^{2}\right)$ and then incubated with the usual medium containing $0.06 \mathrm{mM} \mathrm{Ca}^{2+}$ (control) or with a medium supplemented with $\mathrm{Ca}^{2+}(1.5 \mathrm{~mm})$ for $48 \mathrm{~h}$ before extraction of total RNA.

\section{Brefeldin A incubation}

Confluent monolayers of keratinocytes were incubated with $10 \mu \mathrm{g} /$ $\mathrm{ml}$ brefeldin A for $30 \mathrm{~min}$.

\section{Reconstruction of human epidermis and processing for} histology

Reconstructed human epidermis were produced and analysed as described (20), except fixation in $70 \%$ ethanol $(100 \%), 10 \%$ formalin and 5\% glacial acetic acid.

Human normal mouth epithelium, abdominal skin and thymus sections

A lip fragment was obtained thanks to Pr. Pierre Garin and Alain Koninckx (Laboratoire d'anatomie, University of Namur, Namur, Belgium) from two individuals at autopsy. Abdominal skins were collected from plastic surgery (Dr. B. Bienfait, Clinique St. Luc, Namur, Belgium). A normal thymus fragment was obtained from a young child undergoing corrective cardiovascular surgery for congenital cardiomyopathy. Tissues were embedded in OCT compound, and serial cryosections of 6-8 $\mu \mathrm{m}$ thick were obtained. Immunofluorescence and immunoperoxidase labelling on tissues and cells

Protocols are available in the Supplementary Material.

\section{Real-time RT-PCR for keratinocyte monolayers and} reconstructed human epidermis

Total RNA of monolayers was isolated using High Pure RNA isolation kit (Roche, Basel, Switzerland), according to manufacturer's instructions. Total RNA of reconstructed human epidermis was isolated using RNeasy mini kit (Qiagen, Hilden, Germany), using spin technology for animal cells according to manufacturer's instructions. $1 \mu \mathrm{g}$ of total RNA was reverse transcribed using SuperScript II kit (Life technologies, Carlsbad, California, USA), according to the manufacturer's instructions. Amplification reaction assays contained FastStart Universal SYBR Green Master (Rox) (Roche) and $300 \mathrm{~nm}$ of primers for real-time PCR. Primer sequences are available in the Supplementary Material. The geometric mean of RPLP0 and TBP house-keeping genes values was used for normalization (21). mRNA expression level was quantified using the threshold cycle method on a 7300 real-time PCR machine (Life technologies).

\section{Epidermal dissociation}

Four fractions (T1, T2, T3 and T4) were obtained from normal human abdominal epidermis after successive dissociations of keratinocytes by serial incubations in trypsin-EDTA, as described in (6). Total RNA was extracted from fractions using the RNeasy extraction kit (Qiagen). Three hundred ng of total RNA of each fraction was reverse transcribed using a mixture of dNTPs and random hexamer primers. Amplification assays were performed with the ABI prism 7000 Sequence Detection System and analysed with the corresponding software (Life technologies) using the Maxima Probe/ROX qPCR master mix (Thermo Fisher Scientific, Waltham, MA, USA). Fluorescence was quantified as threshold cycle values. Primer sequences are available in the Supplementary Material. mRNA levels were normalized to HPRT1 house-keeping gene expression levels. This work was performed on skin from two different donors.

\section{Statistical analysis}

Independent experiments were performed at least in triplicates except for mRNA analysis of epidermal dissociation. Statistical significance was evaluated by one-way repeated-measures analysis of variance with a Holm-Sidak correction, except for analysis of calcium-induced differentiation for which we used the paired $t$-test. Data values were expressed as relative quantification level with error bars representing 95\% confidence intervals.

\section{Results}

TMEM45A is upregulated during keratinocyte differentiation in vitro

In autocrine cultures of human epidermal keratinocytes, confluent cells lose their clonogenicity (22) and initiate a limited differentiation programme, as shown by the expression of early and late markers (19). Relative levels of TMEM45A mRNA were quantified at different time points of culture, before and after confluence (Fig. 1a). A very highly significant increase in TMEM45A expression was observed after confluence with an expression profile similar to the differentiation markers keratin-10 (KRT10) and involucrin (IVL). The TM protein 45A expression was then analysed with an affinity-purified peptide antibody as already described (13). The specificity of this antibody was first further validated using competition with the immunogenic peptide (Figure S1). Then, the transmembrane protein $45 \mathrm{~A}$ was labelled in autocrine 

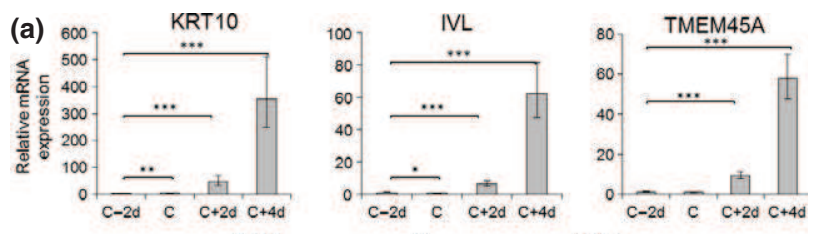

(b)
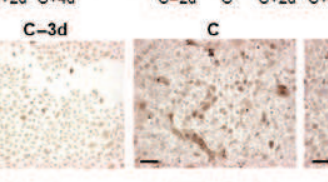

$\mathrm{C}+2 \mathrm{~d}$
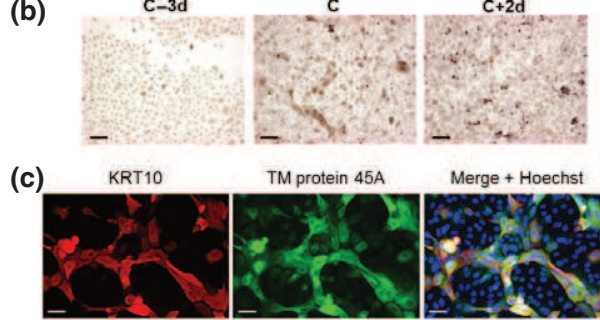

(c)

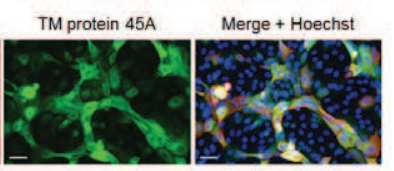

IVL

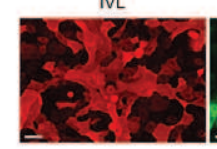

TM protein $45 \mathrm{~A}$

Merge + Hoechst

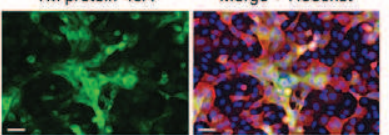

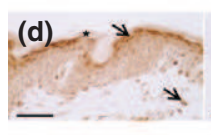

(h) KRT14

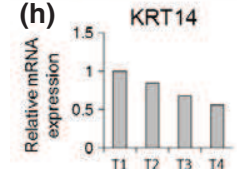

(e)

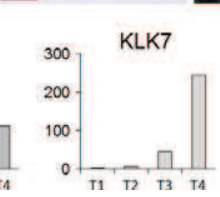

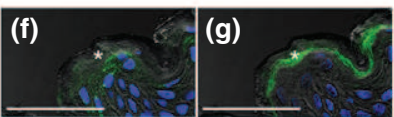

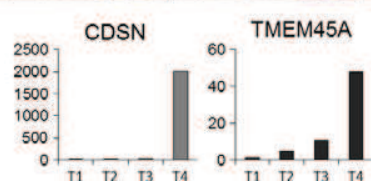

Figure 1. Upregulation of TMEM 45 A during confluence-induced differentiation of keratinocyte autocrine monolayers and predominance of TMEM45A mRNA/protein accumulation in the granular layer of human epidermis. (a) Relative quantification of TMEM45A mRNA 2 days before confluence (C-2d), at confluence (C), 2 days $(C+2 d)$ and 4 days after confluence $(C+4 d)$. Results were normalized to geometric mean values of RPLPO and TBP house-keeping genes. The ' $C-2 d$ ' condition level was arbitrarily fixed at 1. Error bars represent $95 \%$ confidence intervals ( $n=3$, was arbitranily fixed at 1. Error bars represent 95\% confidence intervals $(n=3$, one-way repeated-measures ANOVA, $* P<0.05, * * P<0.01, * * * P<0.001)$. (b) Immunoperoxidase labelling of transmembrane protein 45A 3 days before confluence $(C-3 d)$, at confluence $(C)$ and 2 days after confluence $(C+2 d)$. Hemalun staining of nuclei (scale bars: $100 \mu \mathrm{m}$ ). (c) Co-immunofluorescence labelling of transmembrane protein 45A with KRT10 or IVL 4 days after confluence. Nuclei were stained with Hoechst (scale bars: $50 \mu \mathrm{m}$ ). (d, e) Immunoperoxidase labelling of TM protein 45A in skin. Hemalun staining of nuclei (scale bars: $50 \mu \mathrm{m}$ ). Epidermis and dermal cells show immunoreactivity (arrows). Asterisks indicate cornified layer. Negative control was incubated without primary antibody (e). (f, g) Immunofluorescence labelling of TM protein 45A in skin. TO-PRO-3 staining of nuclei. Pictures show the same field in different Z-planes (scale bars: $50 \mu \mathrm{m})$. (h) Relative mRNA levels of KRT14, KLK7, CDSN and TMEM45A genes in T1, T2, T3 and T4 fractions obtained by trypsin dissociation of epidermal keratinocytes. mRNA levels were normalized to HPRT1 house-keeping gene. The level in T1 was fixed at 1. Results from one representative experiment $(n=2)$.

cultures of keratinocytes at subconfluence, confluence and postconfluence (Fig. 1b). Our data illustrate that immunoreactivity is found at each stage and increases at culture confluence and later. Concomitant detections of the TM protein 45A and both of KRT10 and IVL in differentiating keratinocytes were also established using immunofluorescence co-labelling after culture confluence (Fig. 1c). To analyse whether TMEM45A gene expression is related to differentiation rather than to the high cell density obtained at confluence, its mRNA levels were measured after induction of differentiation by an increased $\mathrm{Ca}^{2+}$ concentration in culture medium. Keratinocytes at low cell density were cultured with a high $(1.5 \mathrm{~mm})$ or low (0.06 mM) calcium concentration for $48 \mathrm{~h}$. A very highly significant increase in KRT10 and IVL expression confirmed the commitment towards differentiation induced by high calcium concentration in the medium (Figure S2). Simultaneously, TMEM45A expression was also significantly increased, further demonstrating its link with epidermal differentiation.

TMEM45A is predominantly expressed by the most differentiated living keratinocytes of the epidermis and is related to keratinization

TM protein $45 \mathrm{~A}$ immunoreactivity (Fig. $1 \mathrm{~d}$, e) was found in all epidermal layers, in fibroblasts and skin appendages (data not shown) in normal human abdominal skin; however, the strongest signal in epidermis was observed in the granular layer. Weaker signals were noticed in basal and spinous layers. To overcome the limited resolution of chromogenic immunohistochemistry, observations using confocal microscopy were performed after immunofluorescence labelling (Fig. 1f, g). In granular layer, a punctated cytoplasmic signal was observed, whereas a rather lamellar signal was localized in the lower part of the cornified layer. Furthermore, TMEM45A relative mRNA levels were quantified in the four fractions of enriched keratinocytes (T1, T2, T3 and T4) obtained after serial incubations of epidermal fragments with trypsin solution (6). T1 fraction was enriched in undifferentiated keratinocytes and T4 fraction in keratinocytes from granular and cornified layers; T2 and T3 contained keratinocytes with an intermediate phenotype. This experiment was performed twice with epidermis from different donors (one representative experiment is shown in Fig. 1h). KRT14 as a marker of basal keratinocytes together with kallikrein 7 (KLK7) and corneodesmosin (CDSN) as markers of granular cells were used as internal controls to assess successful enrichment. The highest relative TMEM45A mRNA level was observed in T4, confirming that TMEM45A expression is predominant in differentiating keratinocytes.

A model of epidermal reconstruction in vitro was then used to follow TMEM45A expression during morphogenesis and keratinization in culture (20). No statistically significant variation in TMEM45A relative mRNA level was observed during morphogenesis (Figure S3a), indicating that TMEM45A transcript abundance increases concomitantly with the amount of transcripts encoded by house-keeping genes during tissue reconstruction. The immunoreactivity for transmembrane protein $45 \mathrm{~A}$ (Figure S3b) was detected in suprabasal (spinous and granular) layers, from 3 days of reconstruction on, when corneocytes appear at the air-liquid interface.

To determine whether TM protein $45 \mathrm{~A}$ accumulation in stratified epithelia relates to keratinization, immunohistochemical detection of the protein was performed on samples of human normal mouth epithelia from two different donors. In parallel to the absence of keratinization in this epithelium, no TM protein $45 \mathrm{~A}$ immunoreactivity was found in suprabasal layers, conversely to the epidermis (Fig. 2a-d). These data indicate that, in non-keratinized keratinocytes, TMEM45A expression is not enhanced like in the epidermis. Interestingly, TMEM45A expression was analysed in one sample of normal human thymus, where epithelial cells undergo keratinization as in epidermal keratinocytes thereby forming Hassal bodies. Accordingly, TM protein 45A immunoreactivity was indeed restricted to Hassal bodies (Fig. 2e). Co-detections of TM protein 45A with KRT14, KRT10 or FLG in the thymus revealed that TM protein $45 \mathrm{~A}$ signal was detected in association with KRT10 and FLG in Hassal bodies only, whereas it 
(a)

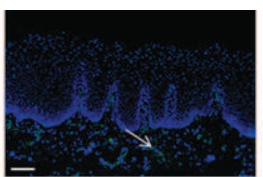

(b)

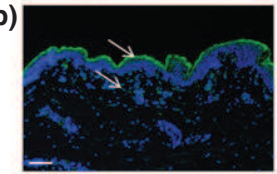

(e)


TM protein 45A

(2) (c)

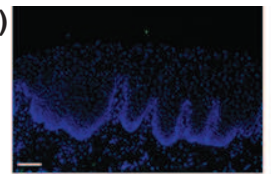

(d)
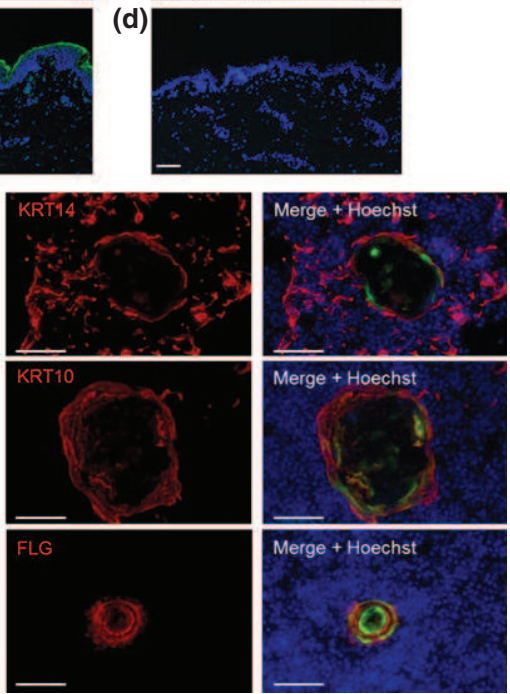

Figure 2. TM protein $45 \mathrm{~A}$ accumulates in thymic Hassal bodies, but is not detected in non-keratinized stratified epithelium. Immunofluorescence labelling of TM protein 45A in normal human non-keratinized mouth epithelium $(a, b)$ and in normal human skin $(c, d)$. Negative controls were produced by incubation without primary antibody ( $b$ and d). Nuclei were stained with Hoechst 33258 (scale bars: $100 \mu \mathrm{m})$. Keratinocytes and fibroblasts show immunoreactivity (arrows). (e) Immunofluorescence labelling of TM protein 45A, KRT14, KRT10 and FLG in thymus. Nuclei were stained with Hoechst 33258 (scale bars: $50 \mu \mathrm{m}$ ).

was surrounded by KRT14 localized outside keratinized Hassal bodies as described (23) (Fig. 2e).

TM protein $45 \mathrm{~A}$ partially accumulates in the trans-Golgi/ trans-Golgi network of keratinocytes and does not reach the lysosomal compartment

The subcellular localization of TM protein 45A was analysed using confocal microscopy. In keratinocytes grown as monolayers, TM protein $45 \mathrm{~A}$ immunoreactivity was located in the cytoplasm, with a mostly perinuclear distribution (Fig. 3a, b). Because TM protein $45 \mathrm{~A}$ is a putative transmembrane protein, its location was compared to specific markers of the endomembrane system. CALN, GM130 and golgin-97 were investigated, respectively, as a molecular chaperone of the endoplasmic reticulum (24), as a cis-Golgi/ cis-Golgi network matrix protein and as a trans-Golgi/trans-Golgi network protein (25) in cultured keratinocytes (Fig. 3a). No colocalization of TM protein 45A with CALN was observed, whereas frequent but partial co-localization with golgin-97 was found, indicating the likely localization of TM protein $45 \mathrm{~A}$ in transGolgi/trans-Golgi network. Interestingly and in perfect accordance with a localization of TM protein $45 \mathrm{~A}$ in trans-Golgi compartment, the TM protein $45 \mathrm{~A}$ signal was often interlocked with GM130 immunoreactivity, suggesting that TM protein $45 \mathrm{~A}$ is localized in an organelle intimately associated with the cis-Golgi/ cis-Golgi network compartment. The same results were obtained at all cell densities (data not shown). To confirm a relationship between TM protein 45A and the Golgi apparatus, brefeldin A was used to interfere with the structural organization of this organelle (Fig. 3b). As expected, CALN localization was unaffected by (a)
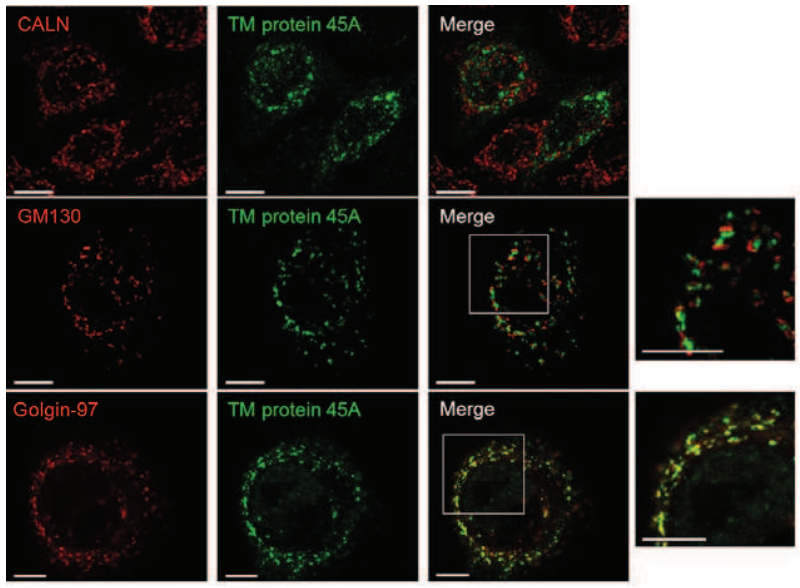

(b) Control
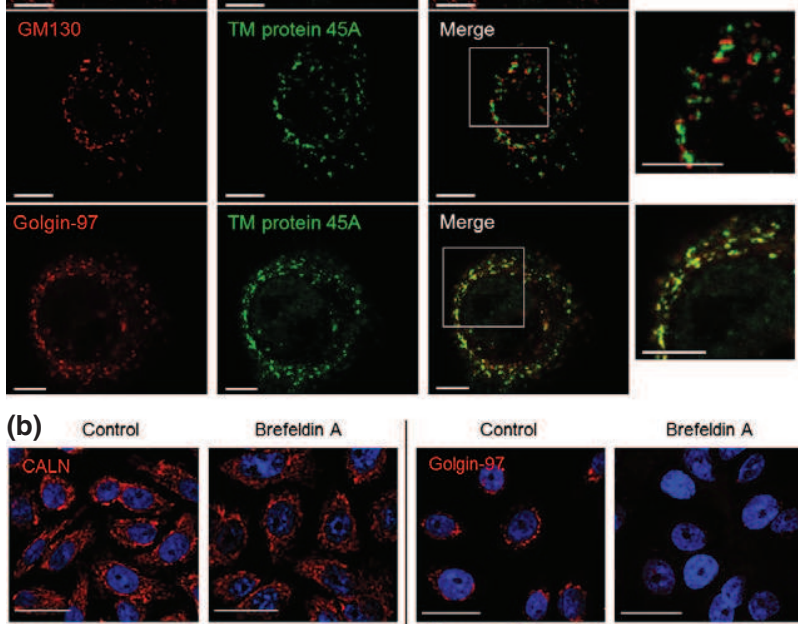

Brefeldin $A$

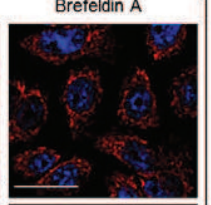

Control

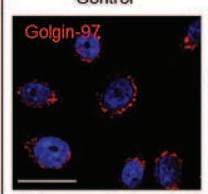

Brefeldin A


Figure 3. Association of TM protein $45 \mathrm{~A}$ with the Golgi apparatus in normal human epidermal keratinocytes. (a) Immunolabelling of TM protein 45A, CALN, GM130 and golgin-97 (scale bars: $10 \mu \mathrm{m}$ ). (b) Immunolabelling of CALN, GM130, golgin-97 and TM protein 45A of confluent cultures incubated without (control) or with brefeldin A $10 \mu \mathrm{g} / \mathrm{ml}$ for $30 \mathrm{~min}$. Nuclei were stained with TO-PRO-3 (scale bars: $25 \mu \mathrm{m})$.

incubation with brefeldin $\mathrm{A}$, whereas the localization of both GM130 and golgin-97 was disrupted. In accordance with association of TM protein $45 \mathrm{~A}$ with the Golgi apparatus, the localization of TM protein $45 \mathrm{~A}$ was disrupted by brefeldin $\mathrm{A}$.

To confirm this result in vivo, co-detections of TM protein 45A with GM130 and golgin-97 were also performed on histological sections of normal human skin (Fig. 4a). TM protein 45A signal was mostly located in granular layer of the epidermis. There, TM protein $45 \mathrm{~A}$ was often localized side by side and alternating with GM130. However, it was not associated with GM130 in more superficial locations. This seems to correspond to its presence in cornified keratinocytes. As observed in cultured cells, TM protein 45A also co-localized, although partially, with golgin-97 in granular keratinocytes.

Thus, association of TM protein $45 \mathrm{~A}$ with the trans-Golgi/ trans-Golgi network was demonstrated, but as this was not its only location, a potential association with the lysosomal compartment was also investigated through co-detection with LAMP1 in cultured keratinocytes (Figure S5a). Using a validated antibody against LAMP1 (26), no co-localization was observed, indicating that TM protein $45 \mathrm{~A}$ does not reach the lysosomal compartment. Ultrastructural analysis of epidermal TM protein 45A Immunogold-labelling of TM protein $45 \mathrm{~A}$ was used to further determine its location in normal epidermis by electron microscopy. 
(a)

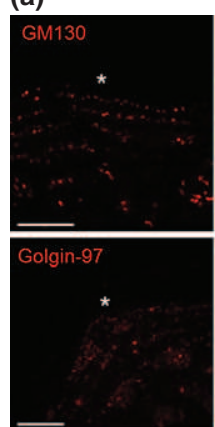

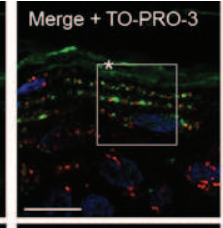

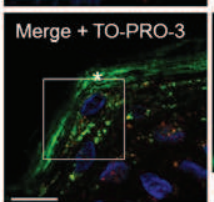

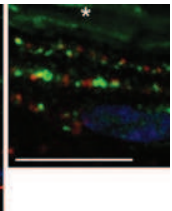

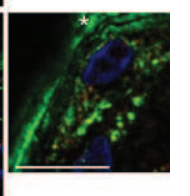

(b)

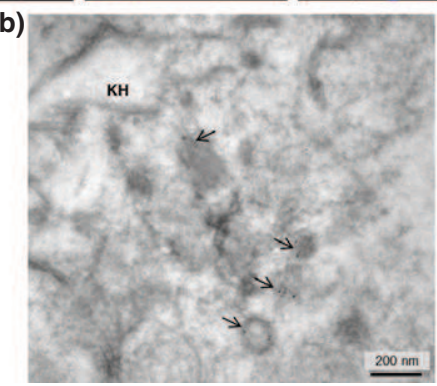

Figure 4. Transmembrane protein $45 \mathrm{~A}$ localization in epidermis. (a) Immunohistochemical staining of GM130, golgin-97 and TM protein 45A in human epidermis. Nuclei were stained with TO-PRO-3. The asterisks indicate the cornified layer. The right panel is a magnification of the merge (scale bars: $10 \mu \mathrm{m})$. (b) layer. The right panel is a magnification of the merge (scale bars: $10 \mu \mathrm{m}$ ). $(\mathrm{b})$
Postembedding immuno-electron microscopy of normal human epidermis using Lowicryl K4M resin and $5 \mathrm{~nm}$ immunogold-labelled secondary antibody. The picture illustrates a granular keratinocyte with keratohyalin granule $(\mathrm{KH})$. Arrows indicate labelled membrane vesicles.

In granular keratinocytes, a high number of gold particles were detected in small intra-cellular membrane vesicles (Fig. 4b). Some labelling was also observed associated with intermediate filaments in the vicinity of keratohyalin granules (Fig. 4b), as well as close to desmosomes (Figure S4a). Inside corneocytes, gold-labelling was dispersed, sometimes associated with anchorage sites of intermediate filaments to corneodesmosomes (Figure S4b). Those localizations concur with the immunofluorescence labelling shown on Fig. 1d-g.

TM protein $45 \mathrm{~A}$ is not transported with corneodesmosin in lamellar bodies

Several observations indicate that TM protein $45 \mathrm{~A}$ could be related to lamellar bodies with namely a punctated distribution in granular layer, a predicted transmembrane localization of the protein, a possible involvement during keratinization, its detection in trans-Golgi/trans-Golgi network and its ultrastructural detection in particular vesicles. To test this hypothesis, co-detection of TM protein 45A was performed in normal epidermis with CDSN, one of cargoes of lamellar bodies, using a validated antibody (27). No or very scarce co-localization could be observed in the granular layer (Figure S5b), indicating that TM protein $45 \mathrm{~A}$ is likely not transported with CDSN in lamellar bodies. However, because cargoes are known to be transported as distinct aggregates (28), this result does not exclude potential relationship of TM protein $45 \mathrm{~A}$ with lamellar bodies.

\section{Discussion}

This study confirms that TMEM45A expression in human keratinocytes, although present in subconfluent undifferentiated

keratinocytes, is upregulated during epidermal differentiation, either induced by culture confluence or enhancement of $\mathrm{Ca}^{2+}$ concentration in medium, as previously published $(13,14)$. It also demonstrates strong connection between TMEM45A expression and keratinization, in both epidermis in vivo or during reconstruction in culture, but also in Hassal bodies inside the thymus. TMEM45A gene expression is increased in parallel with differentiation in cultured keratinocytes and its protein product accumulates during epidermal morphogenesis in vitro. Finally, although present in basal and spinous layers, TM protein 45A accumulation culminates in the granular layer in vivo. Altogether, these data establish TMEM45A expression as a late marker of epidermal differentiation and suggest that it could be an actor of keratinization. These results are consistent with published literature identifying TMEM45A gene as a novel human keratinization-associated gene (15), strongly expressed in late stage of keratinization (7) and as a novel gene selectively and highly expressed in normal human skin (14). This study allows to compile dispersed, but important findings about the link between TMEM45A expression and keratinization that still requests further investigation.

TMEM45A mRNA was found upregulated in psoriasis and actinic keratosis $(14,16)$. In both epidermal pathologies, proliferation and differentiation processes of keratinocytes are disrupted, leading to hyperplasia, hyperkeratosis and parakeratosis (29-32). As these pathologies are characterized respectively by the loss and thinning of the granular layer, results showing upregulation of TMEM45A expression in these conditions were unexpected (30,33). Moreover, TMEM45A was found downregulated in cutaneous squamous cell carcinoma in comparison with actinic keratosis (17), a frequent precursor lesion of squamous cell carcinoma in humans (32). This result could suggest that TMEM45A expression is not required for progression and invasion of cancerous keratinocytes. In breast cancer cells, the gene was shown suppressing progression of ductal carcinoma into invasive breast cancer (18). However, in another study on breast and liver cancer cells, TMEM45A expression was shown essential for hypoxia-induced resistance against drug-induced apoptosis (13). Potential deregulations or implications of TMEM45A in skin pathologies and cancers regarding its association with keratinization need to be further investigated.

Other members of the heterogeneous TMEM family are still poorly characterized, but new findings show potential importance in epidermis functionality or in chemoresistance of cancer cells. Tmem79 (Matt) gene is linked to Golgi apparatus and to lamellar body secretion; altered cornified barrier formation and dermatitis are observed in Tmem $79^{\mathrm{ma} / \mathrm{ma}}$ mutant mice (34). Transmembrane protein 205 is associated with drug resistance of squamous carcinoma, probably by altered membrane trafficking and secretion (35).

For the first time, TM protein 45A precise subcellular localization is reported in keratinocytes. It partially accumulates in transGolgi/trans-Golgi network, both in vitro and in the granular layer in vivo, in perfect accordance with its localization close to cisGolgi/cis-Golgi network. Moreover, its localization is disrupted by brefeldin A, supporting strong relationship with Golgi apparatus. However, other localizations may be suspected, whereas our data exclude lysosomes as well as CDSN-containing lamellar bodies. Nevertheless, transport with other cargoes cannot be excluded. 
Using electron microscopy, TM protein 45A immunoreactivity was associated with membrane vesicles in granular keratinocytes. The signal was also found at the level of insertion sites of intermediate filaments in the vicinity of keratohyalin granules and desmosomes in the granular layer, as well as in anchorage sites of intermediate filaments to corneodesmosomes in the cornified layer. This result suggests a possible association with cytoskeleton.

In conclusion, TMEM45A/transmembrane protein 45 , linked to Golgi apparatus, is associated with epidermal keratinization. This strong relationship indicates the relevance of TMEM45A in this process. Additional functional studies are currently performed to get more insight into its roles.

\section{Acknowledgements}

AH is a research fellow funded by FRIA. ER is financed by Televie grant (FNRS). The study received also financial support from FNRS and FRFC (contract grants $1.5 .033 .06 \mathrm{~F}$ and $2.4 .522 .10 \mathrm{~F}$ ) to YP. The authors thank Dr. Bernadette Bienfait, Pr. Pierre Garin, Alain Koninckx, Noëlle Ninane, Catherine Demazy, Marc Dieu, Lionel Flamant, Dominique Desnoeck, Benoît Balau, Daniel Van Vlaender, Frédéric Minner and Françoise Herphelin for their help in this study.

\section{Author contributions}

Aurélie Hayez, Yves Poumay, Catherine Lambert de Rouvroit and Carine Michiels designed the study. Aurélie Hayez, Marie Reynier, Edith Roegiers and Marek Haftek performed the research. Guy Serre, Vincent Geenen, Chantal Renard and Michel Simon provided essential materials. Aurélie Hayez, Jérémy Malaisse, Yves Poumay, Catherine Lambert de Rouvroit and Carine Michiels analysed the data and wrote the paper. All the authors gave approval for the submitted version.

\section{Conflict of interest}

The authors have declared no conflicting interests.

\section{References}

Eckhart L, Lippens S, Tschachler E et al. Biochim Biophys Acta 2013: 1833: 3471-3480.

2 McLean W H, Irvine A D. Ulster Med J 2007: 76: $72-82$.

3 Shetty S, Gokul S. Oman Med J 2012: 27: 348357.

4 Hamada $T$, Tsuruta $D$, Fukuda $S$ et al. Exp Dermatol 2013: 22: 83-87.

5 Candi E, Schmidt R, Melino G. Nat Rev Mol Cell Biol 2005: 6: 328-340.

6 Toulza E, Mattiuzzo N, Galliano M-F et al. Genome Biol 2007: 8: R107.

7 Taylor J M, Street T L, Hao L et al. PLoS ONE 2009: 4: e7651.

8 BioGPS. Accessed 27 November 2013; Available from: http://biogps.org/\#goto=genereport\&id= 55076

9 Ensembl. Accessed 27 November 2013; Available from: http://www.ensembl.org/Homo_sapiens/ Gene/Summary?g=ENSG00000181458;r=3:1002 11463-100296288.

10 PredictProtein. Accessed 30 October 2013; Available from: https://www.predictprotein.org/ get_results?req_id $=452078$

11 SOSUI. Accessed 27 November 2013; Available from: http://bp.nuap.nagoya-u.ac.jp/sosui/.

12 Uniprot. Accessed 27 November 2013; Available from: http://www.uniprot.org/uniprot/Q9N WC5.

13 Flamant $\mathrm{L}$, Roegiers $\mathrm{E}$, Pierre $\mathrm{M}$ et al. BMC Cancer 2012: 12: 391.

14 Gerber P A, Hevezi P, Buhren B A et al. PLoS ONE 2013: 8: e63949.
15 Mattiuzzo N R, Toulza E, Jonca $N$ et al. Exp Dermatol 2011: 20: 113-118.

16 Hudson L G, Gale J M, Padilla S R et al. Mo Carcinog 2010: 49: 619-629.

17 Lambert S R, Mladkova N, Gulati A et al. Br Cancer 2014: 110: 520-529.

18 Lee S, Stewart S, Nagtegaal I et al. Cancer Res 2012: 72: 4574-4586.

19 Minner F, Herphelin F, Poumay Y. Methods Mol Biol 2010: 585: 71-82

20 Frankart A, Malaisse J, De Vuyst E et al. Exp Dermato 2012: 21: 871-875.

21 Minner F, Poumay Y. J Invest Dermatol 2009: 129: $770-773$.

22 Poumay $Y$, Pittelkow M R. J Invest Dermatol 1995: 104: 271-276.

23 Hale L P, Markert M L. J Immunol 2004: 172: 617-624.

24 Ireland B S, Niggemann M, Williams D B. Methods Mol Biol 2006: 347: 331-342.

25 Goud B, Gleeson P A. Trends Cell Biol 2010: 20 329-336.

26 Sarafian V, Jans R, Poumay $Y$. Arch Dermatol Res 2006: 298: 73-81.

27 Caubet $C$, Jonca N, Brattsand $M$ et al. J Invest Dermatol 2004: 122: 1235-1244.

28 Ishida-Yamamoto A, Simon M, Kishibe $M$ et al. J Invest Dermatol 2004: 122: 1137-1144.

29 Raut A S, Prabhu R H, Patravale V B. Crit Rev Ther Drug Carrier Syst 2013: 30: 183-216.

30 Tonel G, Conrad C. Int J Biochem Cell Biol 2009: 41: 963-968.
31 Berman B, Cockerell C J. J Am Acad Dermatol 2013: 68(1 Suppl 1): S10-S19.

32 Ratushny V, Gober M D, Hick R et al. J Clin Invest 2012: 122: 464-472.

33 Medscape. Actinic Keratosis Pathology. Accessed 3 March 2014; Available from: http://emedicine. medscape.com/article/1976538-overview

34 Sasaki T, Shiohama A, Kubo A et al. J Allergy Clin Immunol 2013: 132: 1111-1120 e4.

35 Shen D W, Gottesman M M. Pharm Res 2012: 29: 643-650.

\section{Supporting Information}

Additional Supporting Information may be found in the online version of this article:

Figure S1. Validation of anti-transmembrane protein $45 \mathrm{~A}$ antibody by immunofluorescence labelling in human skin.

Figure S2. Upregulation of TMEM45A expression during $\mathrm{Ca}^{2+}$-induced differentiation in cultures of human keratinocytes cultured as monolayers.

Figure S3. TMEM45A expression during epidermal morphogenesis at air-liquid interface on polycarbonate filter.

Figure S4. Transmembrane protein 45A localization in epidermis.

Figure S5. Co-detections of TM protein 45A with LAMP1 in vitro and with CDSN in the granular layer of epidermis.

Supplementary Material. Antibodies, immunogenic peptide, chemicals, primers for real-time-PCR, labellings and immunoelectron microscopy. 


\section{Supplementary figures}
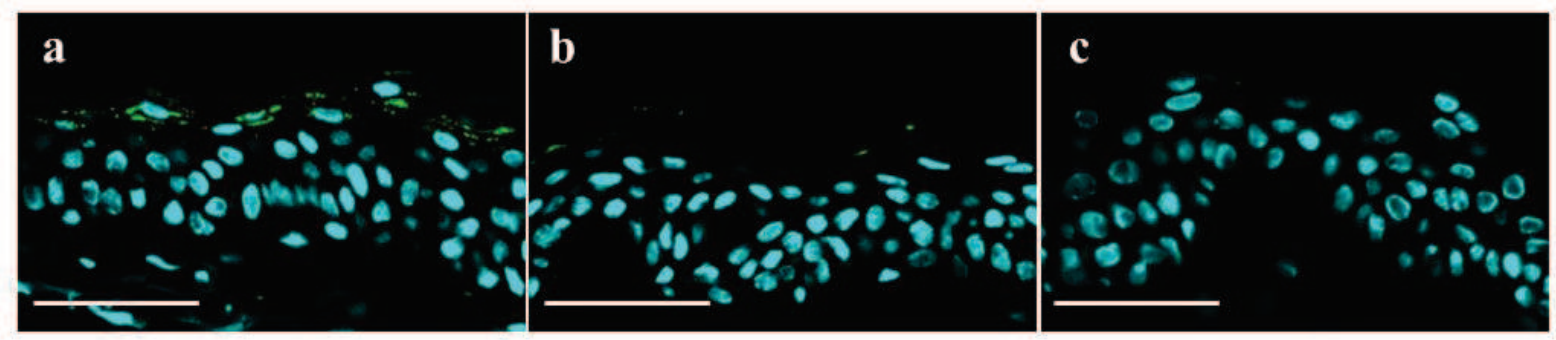

Figure S1. Validation of anti-transmembrane protein $45 \mathrm{~A}$ antibody by immunofluorescence labelling in human skin. Nuclei were stained with Hoechst 33258 (scale bars: $50 \mu \mathrm{m}$ ).

(a) Incubation with primary antibody anti-TM protein 45A was followed by incubation with secondary antibody.

(b) Primary antibody anti-TM protein 45A was incubated with 30 fold excess immunogenic peptide before incubation with tissue. This was followed by incubation with secondary antibody.

(c) Tissue was incubated with secondary antibody only.

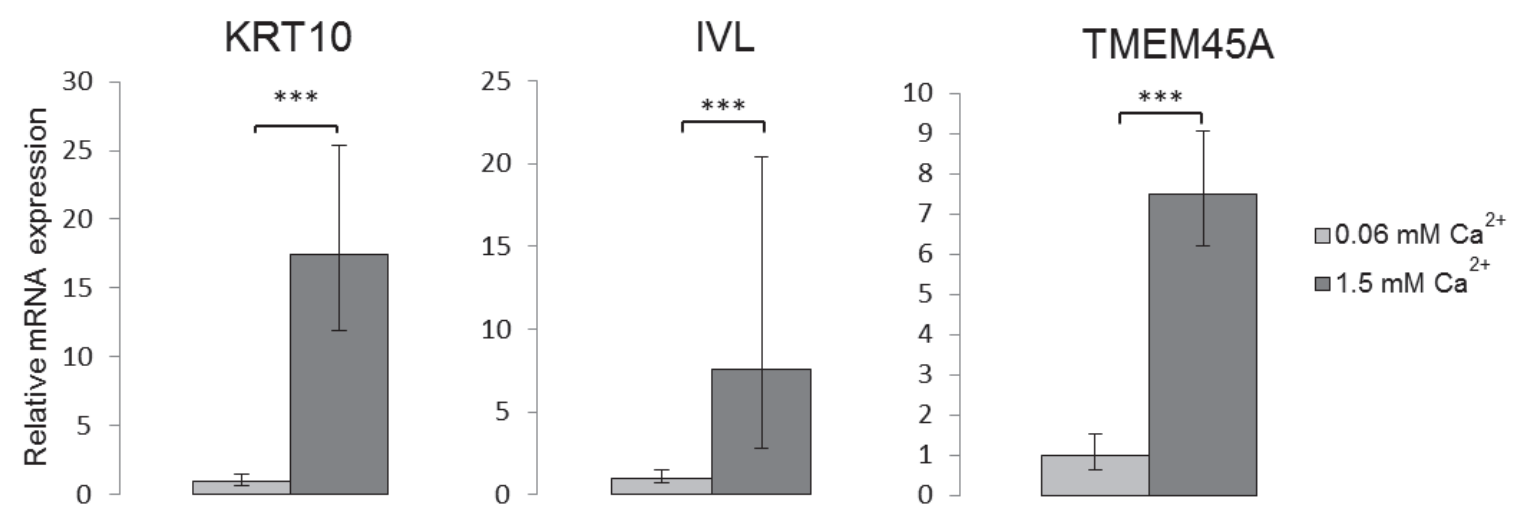

Figure S2. Upregulation of TMEM45A expression during $\mathrm{Ca}^{2+}$-induced differentiation in cultures of human keratinocytes cultured as monolayers.

Subconfluent cultures at low cell density were incubated with high calcium concentration (1.5 $\left.\mathrm{mM} \mathrm{Ca}{ }^{2+}\right)$ during 48 hours. Cultures incubated with the usual calcium concentration $(0.06$ $\mathrm{mM} \mathrm{Ca}{ }^{2+}$ ) were used as reference. mRNA levels were normalized to geometric mean values of two house-keeping genes (RPLP0 and TBP). The mRNA level in the $0.06 \mathrm{mM} \mathrm{Ca}^{2+}$ condition was used as reference and arbitrarily fixed at 1. Error bars represent $95 \%$ confidence intervals $(\mathrm{n}=3$, paired t-test, $* * * \mathrm{P}<0.001)$. 

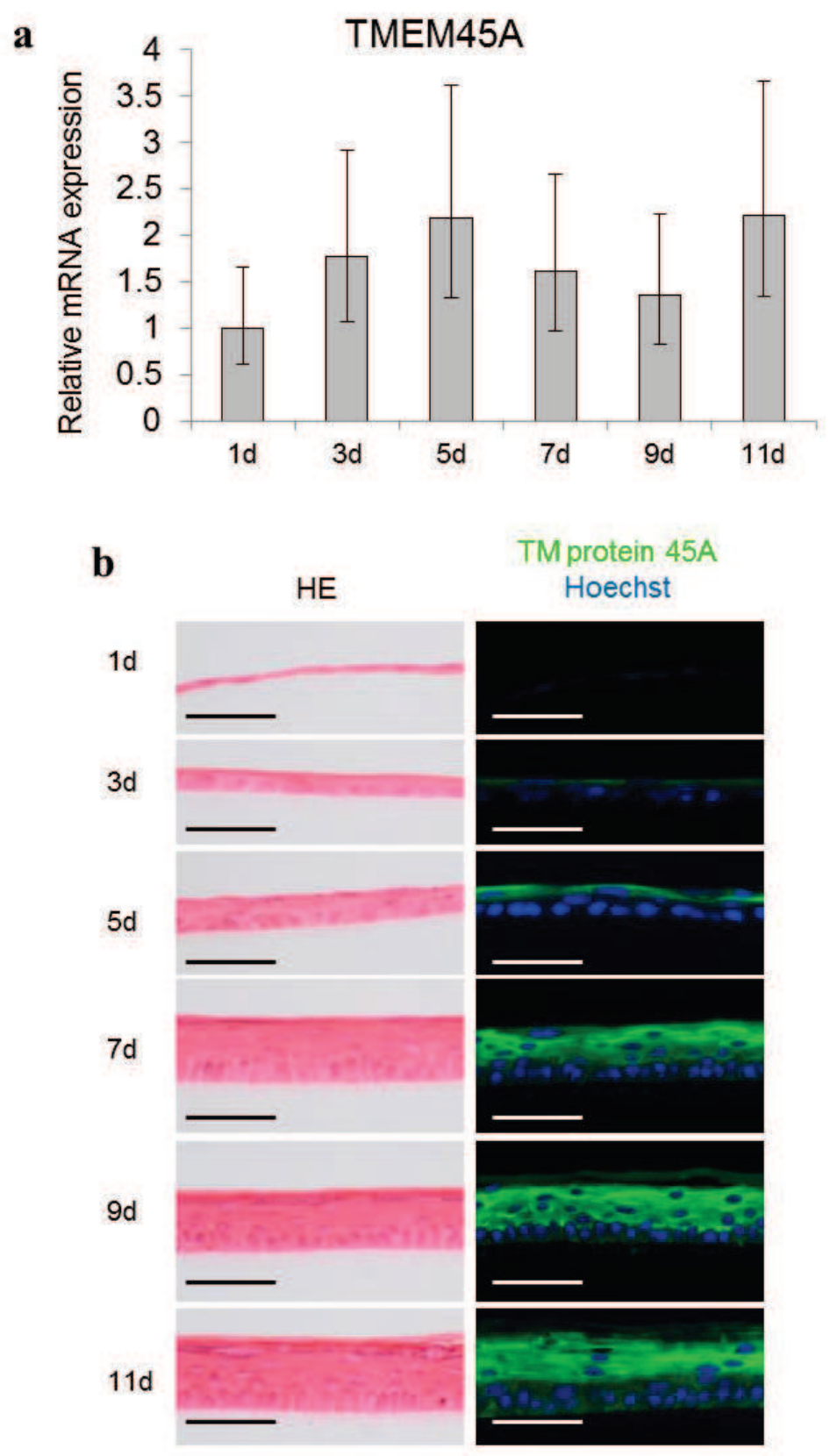

Figure S3. TMEM45A expression during epidermal morphogenesis at air-liquid interface on polycarbonate filter. The reconstructed human epidermis was analyzed one day after seeding the culture (1d), and then every second day until the eleventh day of reconstruction (11d).

(a) mRNA levels were normalized to geometric mean values of TBP and RPLP0 housekeeping genes. Level after one day of reconstruction (1d) was arbitrarily fixed at 1. Error bars represent 95\% confidence intervals ( $\mathrm{n}=4$, one-way Repeated Measures ANOVA). No statistically significant variation was observed.

(b) Immunofluorescent labelling of TM protein 45A. Reconstructed human epidermis stained with Hemalun Erythrosin (HE) or labelled for TM protein 45A detection, followed by DNAstaining using Hoechst 33258 (scale bars: $50 \mu \mathrm{m}$ ). 
a

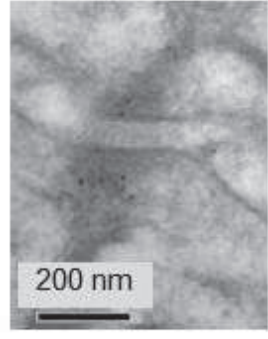

b

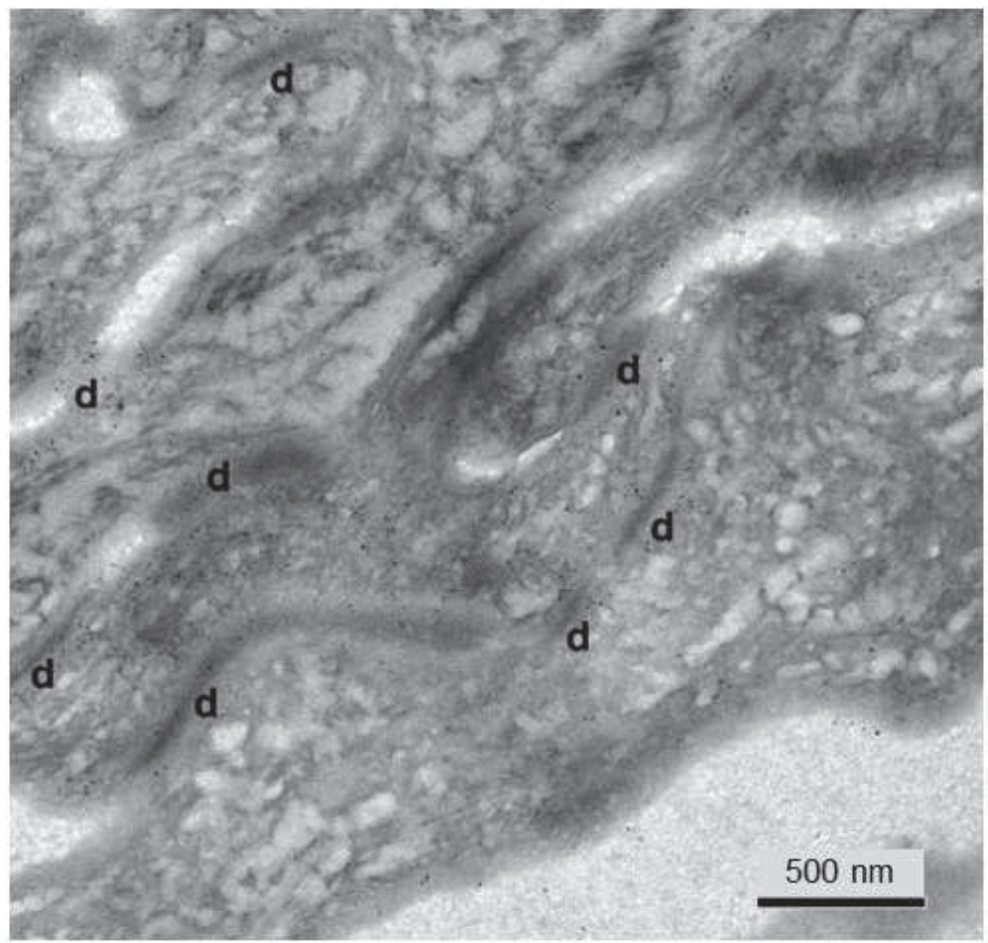

Figure S4. Transmembrane protein 45A localization in epidermis. Post-embedding immuno-electron microscopy of normal human epidermis using Lowicryl K4M resin and 5 $\mathrm{nm}$ immunogold-labeled secondary antibody. (a) Desmosome in the granular layer. (b) Cornified layer with corneodesmosomes (d). 
a

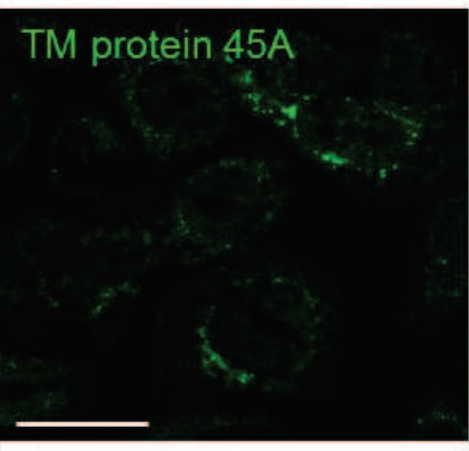

b

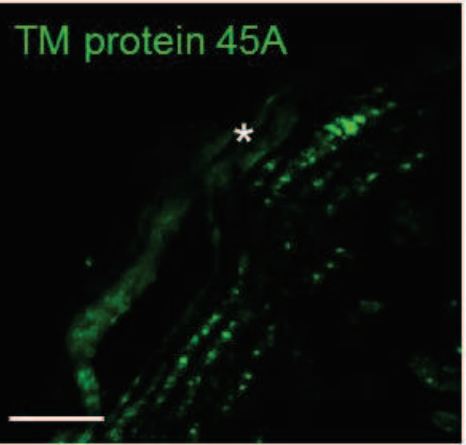

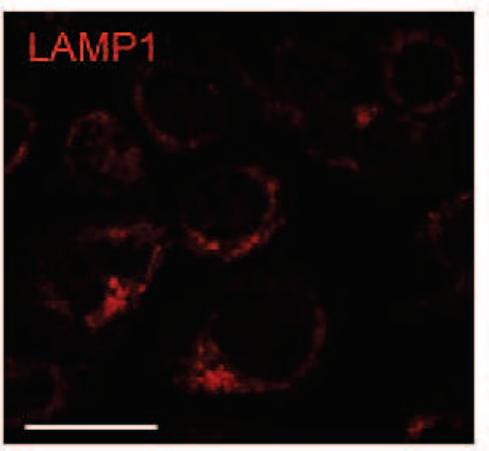

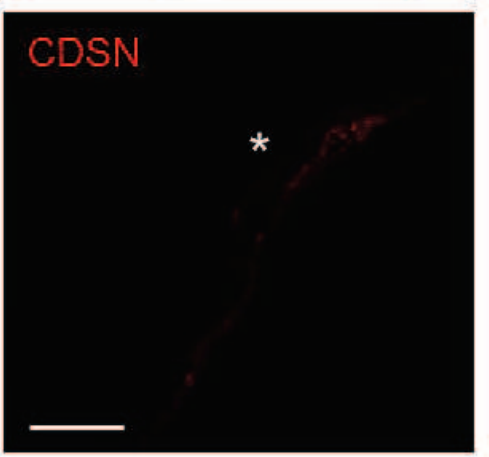



Merge + TO-PRO-3

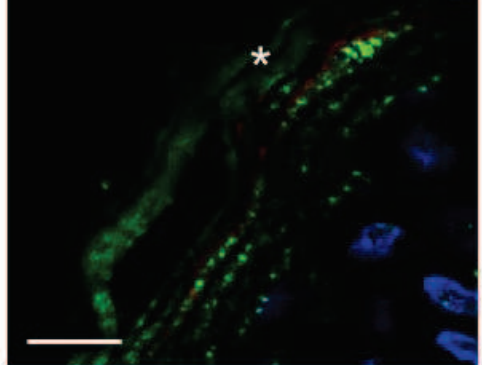

Figure S5. Co-detections of TM protein 45A with LAMP1 in vitro and with CDSN in the granular layer of epidermis.

(a) Immunolabelling of TM protein 45A and LAMP1 in normal human epidermal keratinocytes cultured as autocrine monolayers. Nuclei were stained with TO-PRO-3 (scale bars: $25 \mu \mathrm{m}$ ).

(b) Immunolabelling of TM protein 45A and CDSN in human normal epidermis. Nuclei were stained with TO-PRO-3. The asterisks indicate the cornified layer (scale bars: $10 \mu \mathrm{m}$ ). 


\section{Supplementary material}

\section{Antibodies, immunogenic peptide and chemicals}

Rabbit anti-TM protein 45A antibody $\mathrm{n}^{\circ}$ HPA024082 (dilution 1:50 or 1:100), mouse antiIVL antibody $\mathrm{n}^{\circ} \mathrm{I} 9018$ (dilution 1:200) and brefeldin $\mathrm{A} \mathrm{n}^{\circ} \mathrm{B} 7651$ were purchased from Sigma-Aldrich (Saint-Louis, MO, US). Mouse anti-GM130 antibody $\mathrm{n}^{\circ} 610822$ (dilution 1:100 for cells and 1:300 for tissues) was obtained from BD (Franklin Lakes, NJ, US). Mouse anti-CALN antibody $n^{\circ}$ MA3-027 (dilution 1:500) came from Thermo Fisher Scientific (Waltham, MA, USA). Mouse anti-golgin-97 antibody $\mathrm{n}^{\circ}$ A-21270 (dilution 1:100), Hoechst 33258, TO-PRO-3, Alexa fluor 488-conjugated goat anti-rabbit antibody (dilution 1:200) and Alexa fluor 546-conjugated goat anti-mouse antibody (dilution 1:200 or 1:500) were purchased from Life technologies (Carlsbad, CA, US). Mouse anti-KRT10 antibody $\mathrm{n}^{\circ}$ M7002 (dilution 1:100) and DAB system $n^{\circ}$ K3468 were obtained from Dako (Glostrup, Denmark). Mouse anti-LAMP1 antibody (H4A3) (dilution 1:50) was from DSHB (Iowa City, Iowa, US). Mouse anti-FLG antibody $n^{\circ}$ MS-449-P1 (dilution 1:75) was obtained from Neomarkers (Fremont, CA, US). Mouse anti-KRT14 antibody ${ }^{\circ}$ LL002 (dilution 1:50) was purchased from Santa Cruz (Dallas, Texas, USA). Vectastain ABC Kit peroxidase was purchased from Vector laboratories (Burlingame, CA, US). TMEM45A immunogenic peptide was given by ATLAS ANTIBODIES (Stockholm, Sweden). Mouse anti-CDSN G36-19 antibody (dilution 1:3,000) was given by Guy Serre (UMR5165/U1056 CNRS-INSERMUniversity of Toulouse, UDEAR, Toulouse, France).

Primers for real-time PCR (keratinocyte monolayers and reconstructed human epidermis)

\begin{tabular}{|l|l|l|}
\hline Gene & Forward primer $\left(5^{\prime}{ }^{\prime} 3^{\prime}\right)$ & Reverse primer $\left(3^{\prime}{ }^{\prime}-5^{\prime}\right)$ \\
\hline IVL & TGAAACAGCCAACTCCAC & TTCCTCTTGCTTTGATGGG \\
\hline KRT10 & $\begin{array}{l}\text { AATCA } \\
\text { GATTCTCAACCTAACAAC }\end{array}$ & $\begin{array}{l}\text { CTCATCCAGCA } \\
\text { CCCTACG }\end{array}$ \\
\hline KRT14 & CGATGGCAAGGTGGTGTC & GGGTGAAGCAGGGTCCAG \\
\hline RPLP0 & ATCAACGGGTACAAACGAGTC & CAGATGGATCAGCCAAGAAGG \\
\hline TPB & TCAAACCCAGAATTGTTCTCCTTAT & $\begin{array}{l}\text { CCTGAATCCCTTTAGAATAGGGT } \\
\text { AGA }\end{array}$ \\
\hline TMEM45A & TTATGCAGTAACCATTGTCATCGTT & TGATTCTTGTTCTCGTTCAGCATT \\
\hline
\end{tabular}

Primers for real-time PCR (epidermal dissociation)

\begin{tabular}{|l|l|l|}
\hline Gene & Forward primer $\left(5^{\prime}{ }^{\prime}-3^{\prime}\right)$ & Reverse primer $\left(3^{\prime}{ }^{\prime}-5 '\right)$ \\
\hline KRT14 & GAGGAGACCAAAGGTCGCTA & CTGGATGACTGCGATCCAGA \\
\hline KLK7 & CTCATGCTCGTGAAGCTCAA & GGTCAGAGGGAAAGGTCACA \\
\hline CDSN & CGTATCACCTCCCCTAACGA & AGGAGTAGCTGACCTGGGAA \\
\hline HPRT1 & ACCCCACGAAGTGTTGGATA & AAGCAGATGGCCACAGAACT \\
\hline TMEM45A & TTATGCAGTAACCATTGTCATCGTT & TGATTCTTGTTCTCGTTCAGCATT \\
\hline
\end{tabular}




\section{Immunofluorescence labelling on tissues and cells}

Paraffin-embedded sections were deparaffinized and rehydrated. For TM protein 45A detection, heat-induced antigen retrieval was performed by incubating sections in $10 \mathrm{mM}$ citrate buffer at $\mathrm{pH} 6$ and $95^{\circ} \mathrm{C}$ for 20 minutes. Cryosections and coverslips with seeded cells were fixed for $15 \mathrm{~min}$ in PBS- PFA $4 \%$ buffered at $\mathrm{pH}$ 7.2. After rinsing in $\mathrm{PBS}-\mathrm{CaCl}_{2}$ $(0.01 \%)$, cryosections, paraffin sections and coverslips were incubated in $0.1 \mathrm{M}$ glycine. Paraffin sections were blocked for 30 minutes in PBS-BSA $(0.2 \%)-\mathrm{CaCl}_{2} \quad(0.01 \%)$. Cryosections and coverslips were blocked and permeabilized in PBS-BSA (0.2\%)-Triton X$100(0.02 \%)-\mathrm{CaCl}_{2}(0.01 \%)$ for 30 minutes. Incubation (from 45 minutes to 2 hours or overnight) with primary antibody diluted in PBS-BSA-Triton $\mathrm{X}-100-\mathrm{CaCl}_{2}$ (cryosections and coverslips) or PBS-BSA-CaCl 2 (paraffin sections) was performed. Negative controls were obtained by incubating sections and coverslips in buffer without primary antibody. Sections and coverslips were incubated for one hour with secondary Alexa fluor. Nuclei were stained with Hoechst 33258 or TO-PRO-3. Labelled tissues and cells were analyzed under Olympus AX70 microscope (Tokyo, Japan) or Leica TCS SP5II confocal microscope (Solms, Germany).

\section{Peroxidase labelling on tissues and cells}

Cryosections and coverslips with seeded cells were fixed for 15 minutes in PBS- PFA 4\% buffered at $\mathrm{pH}$ 7.2. After rinsing in $\mathrm{PBS}_{-} \mathrm{CaCl}_{2}(0.01 \%)$, they were incubated in $0.1 \mathrm{M}$ glycine. Cryosections and coverslips were incubated in $3 \% \mathrm{H}_{2} \mathrm{O}_{2}$ for 10 minutes. They were blocked and permeabilized in PBS-BSA (0.2\%)-Triton X-100 (0.02\%)- $\mathrm{CaCl}_{2}(0.01 \%)$ for 30 minutes. Cryosections and coverslips were incubated for 1-2 hours or overnight with primary antibody diluted in PBS-BSA-Triton $\mathrm{X}-100-\mathrm{CaCl}_{2}$. Negative controls were obtained by incubating sections and coverslips in PBS-BSA-Triton $\mathrm{X}-100-\mathrm{CaCl}_{2}$ without primary antibody. Cryosections and coverslips were incubated for 45 min with biotinylated secondary antibody diluted 1:100 in PBS-BSA-Triton X-100. After washes, sections and coverslips were incubated for 30 minutes with streptavidin-peroxidase solution. Detection of HRP was performed using the high-sensitivity DAB system. Reaction was stopped in distilled water. Counterstaining with hemalun was perfomed. For observation, an Olympus AX70 microscope (Tokyo, Japan) was used.

\section{Immunoelectron microscopy}

Normal human skin fixed in 3\% paraformaldehyde/PBS and low-temperature embedded in Lowicryl K4M (Leica, Germany) was used for on-section immunogold labeling [1]. Ultrafine sections, harvested on nickel grids, were first pretreated for $10 \mathrm{~min}$. at $60^{\circ} \mathrm{C}$ with $10 \%$ sodium metaperiodate for surface etching and optimum antigen retrieval. After washes in water and incubation with a blocking buffer containing 5\% normal goat serum, the sections were exposed to the primary antibody diluted $1: 50$, in PBS supplemented with $0.1 \%$ goat serum and $0.01 \%$ gelatin, at $4{ }^{\circ} \mathrm{C}$ overnight. After washes, the primary antibody binding sites were revealed using goat anti-rabbit $5 \mathrm{~nm}$ immunogold-labeled secondary antibody (diluted 1:10, 1h at r.t.; British Biocell International, Cardiff, UK). After final washes, the sections were counterstained with uranyl acetate for observation in TEM. 


\section{Supplementary reference}

1. Haftek, M., et al., Expression of corneodesmosin in the granular layer and stratum corneum of normal and diseased epidermis. Br J Dermatol, 1997. 137(6): p. 864-73. 